\title{
Congenital, Familial and Genetic Disorder Class
}

National Cancer Institute

\section{Source}

National Cancer Institute. Congenital, Familial and Genetic Disorder Class. NCI Thesaurus. Code C49399.

A class of disorders that encompasses conditions resulting from a congenital, familial hereditary trait or genetic abnormality as is manifested by a characteristic set of symptoms and signs. 